\title{
Modulatory Effect of Motivation on the Association of Trait Anxiety and Cognitive Performance: A Pupillometric Study
}

\author{
Takatoshi Hoshino' ${ }^{1}$, Yoshihiko Tanno ${ }^{2}$ \\ ${ }^{1}$ Department of Psychoinformatics, Kanazawa Institute of Technology, Ishikawa, Japan \\ ${ }^{2}$ Graduate School of Arts and Sciences, The University of Tokyo, Tokyo, Japan \\ Email: hoshino@neptune.kanazawa-it.ac.jp
}

How to cite this paper: Hoshino, T. and Tanno, Y. (2017) Modulatory Effect of Motivation on the Association of Trait Anxiety and Cognitive Performance: A Pupillometric Study. Journal of Behavioral and Brain Science, 7, 273-286.

https://doi.org/10.4236/jbbs.2017.77020

Received: March 13, 2017

Accepted: July 9, 2017

Published: July 12, 2017

Copyright $\odot 2017$ by authors and Scientific Research Publishing Inc. This work is licensed under the Creative Commons Attribution International License (CC BY 4.0).

http://creativecommons.org/licenses/by/4.0/

\begin{abstract}
The attentional control theory (ACT) proposes that trait anxiety disrupts functional efficiency of executive control of attention relating to working memory system such as shifting. ACT also emphasizes the modulatory role of motivation in anxiety on cognitive task performance. The present study investigated the association of trait anxiety-related inefficiencies in attentional shift and working memory performance in conjunction with the level of motivation. A variation of complex span paradigm is designed to systematically manipulate the time constraint on shifting attentional focus back and forth between working memory contents and processing task was used in this study. In the experiment, participants high and low in trait anxiety were allocated either high or low motivation induction conditions, and performed a series of complex span tasks. They also completed a state anxiety measure before and after the experimental task. Motivational states were assessed by the pupil dilation, which is known to reflect the amount of cognitive effort invested on the task at hand. Results showed that, only in low motivation condition, high trait-anxious individuals exhibited greater difficulty, relative to those low in trait anxiety, in maintaining working memory contents as the task demand increased. State anxiety showed no relation to working memory performance regardless of the level of motivation and task demands. Pupillary responses revealed that, in high motivational state, high trait-anxious individuals invested more effort than those low in trait anxiety when the task demand was low, $\mathrm{F}(1,132)=6.65, \mathrm{p}=0.0110$, to moderate, $\mathrm{F}(1,132)=8.441, \mathrm{p}=0.00043$. In accordance with $\mathrm{ACT}$, these findings suggest that motivation can modulate the association between trait anxiety and cognitive performance along with the levels of task demand. Clinical implication is briefly discussed.
\end{abstract}

\section{Keywords}

Trait Anxiety, Motivation, Working Memory, Attentional Control Theory 
(ACT), Pupillometry

\section{Introduction}

In current psychological theory, anxiety is conceptualized as two types: trait and state anxiety [1]. Trait anxiety refers to individual differences in the general propensity to experience anxiety, and state anxiety refers to subjectively perceived anxious thoughts, feelings, and heightened physiological arousal in specific situations. A wealth of studies demonstrates that trait anxiety can influence various cognitive processes including higher-order controlled processes as well as early perceptual detection stages. Eysenck and colleagues put forward the cognitive accounts of anxiety-related attentional alteration called attentional control theory (ACT) [2] [3], which posits that elevated levels of trait anxiety decrease the functional efficiency of executive control of attention implemented by the central executive component of working memory (WM) [4] [5], along with the hypervigilance in sensory-perceptual processing. ACT also argues that modulatory effect of motivation on cognitive performance must be considered when examining the association of trait anxiety and cognitive function. According to ACT, high trait-anxious individuals can be more apprehensive about their performance [6], which motivates them to invest more cognitive effort on a task at hand.

ACT can well accommodate much empirical data concerning anxiety-related inefficient executive control over visual-perceptual attention. Pacheco-Unguetti et al. [7] demonstrates that high trait-anxious individuals exhibit difficulty responding to a target arrow flanked with perceptually competing distractor arrows relative to those low in trait anxiety. Similarly, high trait-sanxiou individuals have been shown difficulty performing anti-saccade tasks, in which participants are required to execute a saccade (horizontal eye movement) toward a target stimulus located in the opposite direction of a perceptually salient flashed stimulus [8] [9].

However, whether such anxiety-related in efficiencies in executive control can impact directly on the performance of higher-order cognitive operations such as WM remains unclear with reference to ACT. Some studies demonstrated that high trait-anxious individuals exhibited poorer performance on complex span WM tasks, which are designed to require concurrent processing task while subjects maintain items in WM [10] [11] [12] [13]. Traditional complex span tasks such as the reading span task and the operation span task are administered at the participant's own pace [14] [15]., which makes these findings hard to interpret from the view of "quickness" in attentional sift. Accordingly, whether trait anxiety-related inefficient executive control of attention results directly in reduced WM capacity has not been sufficiently established due to the methodological limitation on strictly controlling temporal parameters.

The Time-Based Resource-Sharing (TBRS) model of WM, which emphasizes 
the temporal factor in WM maintenance [16] [17] is one promising model for examining the association of trait anxiety and WM decrement. The TBRS model accounts for the determinants of performance on complex span task in the following way: 1) subjects are presented with items they must later recall, 2) while maintaining these items in WM, subjects engage in a processing task which require attentional focus, 3) this diversion of attentional resource from memory item results in a time-based decay of the memory traces. As such, performance on complex span task is determined by the temporal constraint on the time spare to direct attention toward the decaying WM items to reactivate them for further retention (i.e., attentional refreshing) [18] [19]. To examine the TBRS model, Barrouillet and colleagues have developed a computer-paced complex span task called continuous processing span task, which is designed to systematically manipulate the time spare to refresh WM items. Thus, the TBRS model defines cognitive load as the proportion of temporal occupation of attentional resources on intervening processing task [16] [17]. This paradigm has yielded a clear-cut linear association between cognitive load and WM maintenance, demonstrating that greater cognitive load should result in poorer WM maintenance (see [20] for a review).Thus far, none of the studies have investigated the impact of trait anxiety-related inefficient executive control on WM performance using a complex span task based directly on the TBRS framework. Given that high trait-anxious individuals would be inefficient in executive control and take more time to shift attention back and forth between a processing task and WM contents, it is predicted that individuals with high levels of trait anxiety would exhibit a difficulty in maintaining WM contents on a continuous processing span task as the cognitive load increased.

Additionally, it is highly important to note that trait anxiety can alter motivational state (and a consequent cognitive effort invested) depending on task goals, as ACT postulates [2] [3]. Hayes et al. [21] investigated the modulatory effect of motivation on the relationship between trait anxiety and cognitive task performance (Experiment 1 and 2). In their experiments, high and low trait-anxious individuals performed intentional (effortful in nature) and incidental (effortless) category learning tasks. Results showed that trait anxiety disrupted the performance on an incidental learning but not an intentional learning. Therefore, the negative impact of anxiety-related inefficient executive control on higher-order cognitive operation should become more apparent in the condition without high motivational state (and resultant extra cognitive effort). Unfortunately, manipulated motivational states or the amount of cognitive effort invested were not measured directly in their study.

In this study, we employed the pupillometry technique to assess participants' motivational states in terms of the amount of cognitive effort invested on the experimental task. It is well documented that pupil dilation is sensitive to participants' motivational states [22]. as well as the task demands and difficulty linked to cognitive effort [23] [24] [25]. If high trait-anxious individuals are more likely to be motivated to perform a demanding task to achieve comparable 
result with those low in trait anxiety in a specific situation where the effort is required, the quantitative differences of cognitive effort should emerge in pupil dilation depending on the level of trait anxiety.

In summary, two predictions derived from ACT were tested. First, in low motivational states, high trait-anxious individuals would perform worse, compared to those low in trait anxiety, on the continuous processing span WM task as cognitive load increased. Secondly, in high motivational states, high trait-anxious individuals would achieve comparable levels of performance, relative to their counterparts, due to anxiety-driven compensatory effort invested on the task (compensatory effort hypothesis). Therefore, they would show greater pupil dilation on performing the task, compared to their counterparts.

\section{Method}

\subsection{Participants}

Forty-eight undergraduate students (age: $18-23$ years, mean age $=19.8,29$ females) participated in the experiment in return for compensation. All participants were the students of The University of Tokyo, Japan, and took second author's introductory psychology course. Information about this study and the request form were sent via e-mail from the first author. They all had normal or corrected-to-normal vision. Based on the previous studies such as [8] [9], they were divided into two groups according to whether their trait anxiety scores were above the median value (high trait-anxiety; HTA; $n=24$ ) or below (low trait-anxiety; LTA; $n=24$ ), as measured by the trait subscale of the State-Trait Anxiety Inventory (STAI) [26] [27]. Written informed consent was obtained from each participant before the experiment. This study was approved by the Ethics Committee of the graduate school of Arts and Sciences, The University of Tokyo.

\subsection{Experimental Task}

The general feature of the continuous processing span task is that the intervening processing task successively follows each memory items. In this experiment, alphabetic consonants were presented one by one at a time as memoranda and a choice reaction time (CRT) task was employed as a processing task.

Participants were seated approximately $60 \mathrm{~cm}$. in front of a 17 -inch monitor. They were presented with a series of letter-strings in ascending order (from 1 to 7 letters). Each length of letter-string was administered three times. For each trial, each letter was followed by a CRT task. In CRT task, a square probe (visual angle, $1.5^{\circ}$ on each side) was presented randomly at $9.5^{\circ}$ on the upper or lower side of a center fixation cross. Participants were required to respond to a probe presented in the upper side by pressing the "L" key and the lower side by the " $\mathrm{A}$ " key. Additionally, we set up three conditions of cognitive load: low, moderate, and high load conditions. Preliminary study revealed that the task required about $398 \mathrm{~ms}$ to make a response. Taking this into account, the time interval for the CRT task (i.e., inter-letter interval) was set for $6500 \mathrm{~ms}$. 
Participants were asked to focus on the fixation cross presented on the center of the screen for $1000 \mathrm{~ms}$ at the beginning of each trial, which was followed by a blank screen for $500 \mathrm{~ms}$. Then, the first letter to be remembered was presented for $1500 \mathrm{~ms}$ and replaced by the fixation cross. After a $500 \mathrm{~ms}$ delay, a square probe was presented either in the upper or lower side from the cross. The probe was displayed for $2000 \mathrm{~ms}$ (low load), $1000 \mathrm{~ms}$ (moderate load) or $500 \mathrm{~ms}$ (high load) and followed by a delay of $1000 \mathrm{~ms}, 500 \mathrm{~ms}$ or $250 \mathrm{~ms}$ (low, moderate or high load) according to the cognitive load condition, which resulted in 2, 4 and 8 response requirements in each processing task sequence. Participants were asked to indicate the position as quickly and accurately as possible by pressing corresponding keys. At the end of a trial, participants were required to recall the letters in serial order. All participants performed 3 series of 1 to 7 letter-strings each. Stimuli were controlled and responses were recorded using E-prime 2.0 software (Psychology Software Tools).

\subsection{Scoring on the Continuous Processing Span Task}

We calculated the Partial-Credit Unit (PCU) as an index of WM performance of the continuous processing span task and the reading span task, based on [28]. The PCU score denotes the mean proportion of correctly recalled items in a serial order. That is,

PCU

$=($ Sum of the proportion of correctly recalled items in a serial order in each trial $) /($ Number of traials $)$

\subsection{Questionnaire}

Trait anxiety levels were measured by the trait subscale of the State-Trait Anxiety Inventory [26] [27]. The state subscale of the STAI (STAI-S) was also used to measure participants' current mood state before and after the experimental task.

\subsection{Pupil Diameter Acquisition}

Pupil diameter on the right eye of each participant was continuously collected at $90 \mathrm{~Hz}$ using View Point Eye Tracker system (Monocular 220 fps; Arrington Research, Inc.) throughout the three conditions of the continuous processing span task. Missing data due to eye blinks were filtered out and interpolated by a spline transformation.

\subsection{Procedure}

In the experimental session, calibration of the eyetracker was first administered. After participants completed the STAI-T, they were randomly allocated either the high or low motivation condition. Participants allocated in the high motivation condition were told that the experimental task they would do was a new intelligence quotient (IQ) test and they had to try it as hard as possible in order to assess their IQ accurately. In contrast, participants allocated in the low motivation condition were told that we researchers were interested in the association of eye movement and memory processes such as encoding and retrieval, not their 
own memory capacity. After a two-minute rest, they filled out the STAI-S which assessed current anxious mood state.

After that, each participant performed the three conditions of the continuous processing span task (low-, moderate-, and high-load conditions) in random order. Before starting the testing trials, they practiced 3 trials with 2 letter-string for each condition to ensure that they comprehended the task requirements. Participants were allowed to repeat the practice if they had any doubt about the task (no participants did). All participants performed 21trials (1 - 7 letters) for each condition. Finally, they again filled out the STAI-S soon after they finished the experimental tasks.

A mixed design Analysis of Variance (ANOVA) was used for examining the effects of Group (HTA v.s. LTA), Condition (high v.s. low motivation), Load (low, moderate, and high cognitive load), and their interactions on the cognitive performance with Group and Condition as between-subject factors and Load as a within-subject factor. Memory Size (the number of to-be-remembered items) was added when comparing participants' motivational states through the pupil responses, as a within-subject factor. Follow-up t-tests were administered with the $\mathrm{p}$-values being Bonferroni-corrected. The level of significance set $\mathrm{p}<0.05$.

\section{Results}

\subsection{Scores on the STAI}

We first analyzed the data from the questionnaires (see Table 1). Regarding the trait anxiety levels, a 2 (Group) $\times 2$ (Condition) two-way ANOVA confirmed that a main effect of Group was significant, $\mathrm{F}(1,44)=14.33, \mathrm{p}=0.00047, \eta^{2}=$ 0.66 , but a main effect of Condition and an interaction were not observed, Fs < 2.42 , ps $>0.125$. The HTA and LTA groups were reliably different from each other for trait anxiety levels.

As for a current state anxiety before the experimental task, a same $2 \times 2$ ANOVA were performed with the scores of the STAI-S as the dependent variable. Importantly, main effects of Group and Condition were significant, $\mathrm{F}(1,44)$ $=6.44, \mathrm{p}=0.0187, \eta^{2}=0.13 ; \mathrm{F}(1,44)=7.34, \mathrm{p}=0.0096, \eta^{2}=0.23$, respectively, but an interaction did not reach significant, $\mathrm{F}(1,44)=3.28, \mathrm{p}=0.077$, suggesting that the manipulation (i.e., cover story of IQ test) successfully induced more ap-

Table 1. Mean scores of trait anxiety (STAI-T) and state anxiety (STAI-S) in the High Trait-Anxiety group and Low Trait-Anxiety group.

\begin{tabular}{|c|c|c|c|c|}
\hline \multirow{3}{*}{ Anxiety Measures } & \multicolumn{4}{|c|}{ Group } \\
\hline & \multicolumn{2}{|c|}{$\begin{array}{l}\text { High Trait-Anxious group } \\
\qquad(\text { HTA } ; \mathrm{n}=24)\end{array}$} & \multicolumn{2}{|c|}{$\begin{array}{l}\text { Low Trait-Anxious group } \\
(\text { LTA; } \mathrm{n}=24)\end{array}$} \\
\hline & Pre-task & Post-task & Pre-task & Post-task \\
\hline STAI-T & $46.7(8.5)$ & - & $33.6(6.7)$ & - \\
\hline STAI-S & $38.2(6.7)$ & $30.6(5.7)$ & $29.3(5.3)$ & $28.9(5.8)$ \\
\hline
\end{tabular}

Standard deviations in parentheses. 
prehension about the performance to participants in high motivation condition at the time of the WM task. After completing the task, no main effects or interaction were observed on the STAI-S scores, Fs $<1.63$, ps $>0.20$.

\subsection{Reaction Times on the Processing Task of the Continuous Processing Span Task}

The mean RTs on the CRT tasks were analyzed with a 2 (Group) $\times 2$ (Condition) $\times 3$ (Load) mixed design ANOVA. A main effect of Load was significant, F (2, $88)=16.37, \mathrm{p}=0.000001, \eta^{2}=0.57$, showing that faster paced presentation resulted in shorter RTs, presumably because the shorter stimulus interval made it easier to sustain attention on the processing task (mean RTs for low, moderate and high load: 414 ms v.s. 393 ms v.s. 356 ms). Main effects of Group and Condition were not significant, F $s<2.50$, ps $>0.122$. None of three- or two-way interactions were observed on RTs, ps $>0.269$. These results indicate that both groups showed comparable efficiency in performing the processing task independently of motivational states. Accuracy rates were fairly high across all participants (mean accuracy rate: $98.7 \%$ ).

\subsection{Working Memory Performance}

The PCU scores were calculated for the index of WM maintenance. Figure 1 shows the mean PCU scores and standard errors of the mean for each experimental group corresponding to cognitive loads. A 2 (Group) $\times 2$ (Condition) $\times 3$ (Load) mixed design ANOVA was performed with PCU as the dependent variable. Main effects of Group, Condition, and Load were significant, $F(1,44)=5.29$,

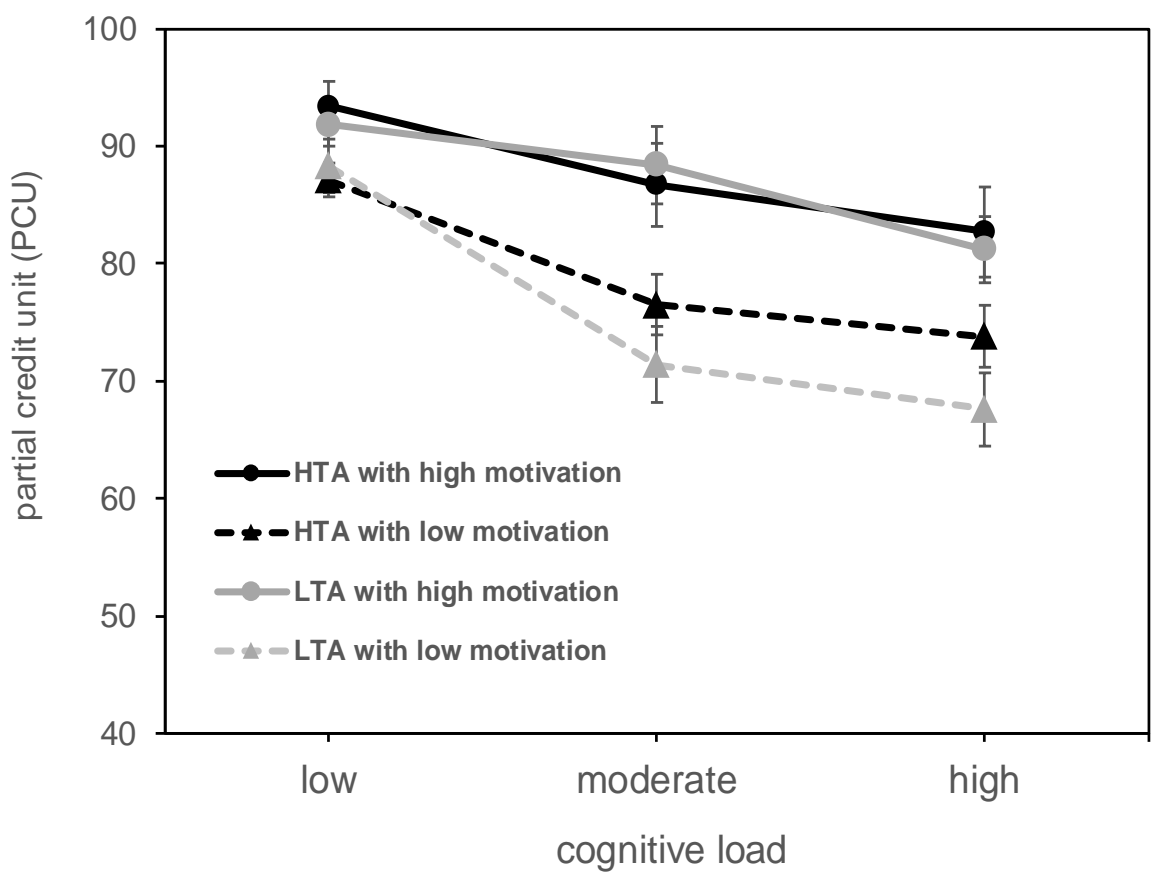

Figure 1. Mean Partial-Credit Unit (PCU) scores in High Trait-Anxiety (HTA) and Low Trait-Anxiety (LTA) groups along with cognitive load and motivational state. Error bars denote standard errors of the mean. 
7.76, 5.94; $\mathrm{p}=0.0221,0.0078,0.0189 ; \eta^{2}=0.12,0.23,0.22$, respectively. As expected, these main effects were qualified by a significant three-way interaction, $\mathrm{F}(2,88)=11.26, \mathrm{p}=0.000044, \eta^{2}=0.41$, indicating that cognitive load on WM maintenance differentially impacted on the HTA and LTA group depending on the motivational states. Additionally, an interaction of Group $\times$ Load did not reach significant, $\mathrm{F}(2,88)=2.23, \mathrm{p}=0.114$, implying the necessity of motivation manipulation for examining the anxiety-cognition relations. Bonferroni-corrected pairwise comparisons between Groups for each Condition and Load confirmed that in low motivation condition the HTA group showed poorer WM performance in the moderate and high load conditions, $\mathrm{t}(46)=2.61, \mathrm{p}=0.0121$; $\mathrm{t}(46)=2.94, \mathrm{p}=0.00512$, respectively, but not in the low load condition, $\mathrm{t}(46)=$ $0.32, \mathrm{p}=0.750$, compared to their counterparts. In high motivation condition, no group differences were detected, ts $<0.64$, ps $>0.525$, indicating the comparable performance of the HTA and LTA group.

Unexpectedly, the relationship between the task performance (PCU scores) and the levels of state anxiety before and after the task were not detected, rs < 0.10 , ps $>0.23$.

\subsection{Motivational States Indexed by Pupil Dilation}

Baseline-corrected pupil dilation was calculated on a trail-by-trial basis. This procedure can eliminate the influence of the carry-over effects from the previous trails [29]. Pupil data collected at the fixation period $(1000 \mathrm{~ms})$ in a trial were averaged for a baseline value of that trail. Then, difference score between the peak and baseline values in that trial was calculated for an index of a (baseline-corrected) pupil dilation [22].

Figures 2(a)-(c) show the changes in baseline-corrected pupil dilation according to levels of trait anxiety and the experimental conditions (i.e., motivational states), on each cognitive load. A 2 (Group) $\times 2$ (Condition) $\times 3$ (Load) $\times$ 7 (Memory Size; the number of to-be-remembered items) mixed design ANOVA was performed with the pupil dilation scores as the dependent variable. In what follows, we shall report results concerning our specific research interest. First, four-way interaction was significant, $\mathrm{F}(12,528)=3.68, \mathrm{p}=0.000024, \eta^{2}=$ 0.41 , indicating that the patterns of changes in pupil dilation along with Group, Condition, and Memory Size were modulated by cognitive load (see Figures $2(\mathrm{a})-(\mathrm{c}))$. A main effect of Condition was significant, $\mathrm{F}(1,44)=6.67, \mathrm{p}=0.0132$, $\eta^{2}=0.30$. Simple effect tests indicated that three simple main effects of Condition in low, moderate, and high load were also significant, $\mathrm{F}(1,132)=6.41$, $14.91,9.25 ; \mathrm{p}=0.0125,0.000174,0.0028$, respectively, suggesting that participants allocated in high motivation condition constantly invested more cognitive effort, compared to those low in motivation, indexed by pupil dilation at any load. As for the compensatory effort hypothesis in anxiety, simple effect of Group in high motivation was significant, $F(1,44)=8.87, \mathrm{p}=0.0047$, but not in low motivation, $\mathrm{F}(1,44)=0.79, \mathrm{p}=0.382$, suggesting that, in high motivational states the HTA group was more effortful on performing the WM task, compared 


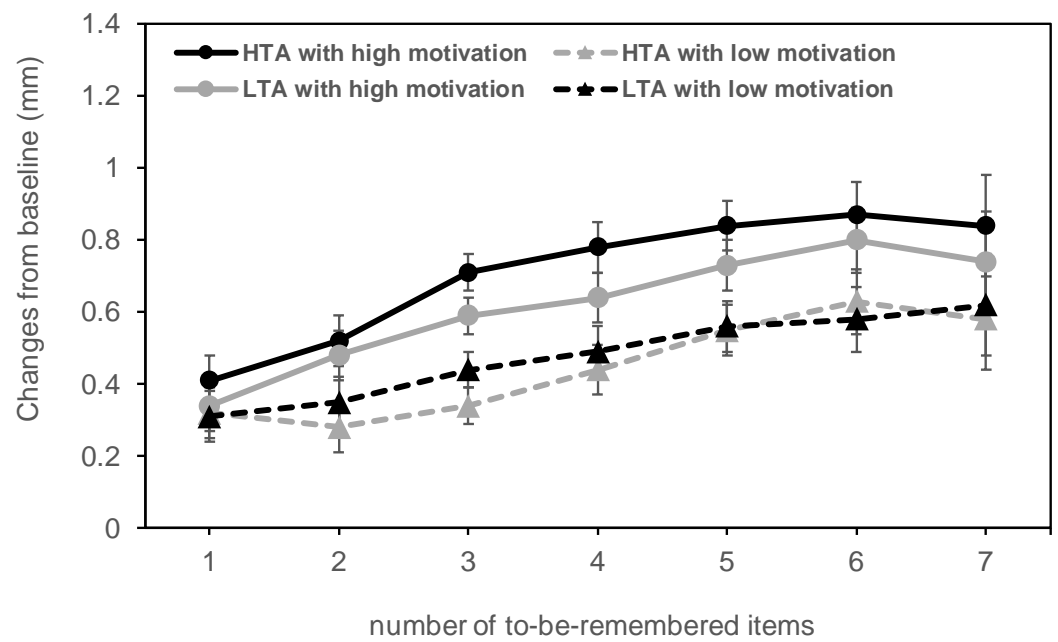

(a)

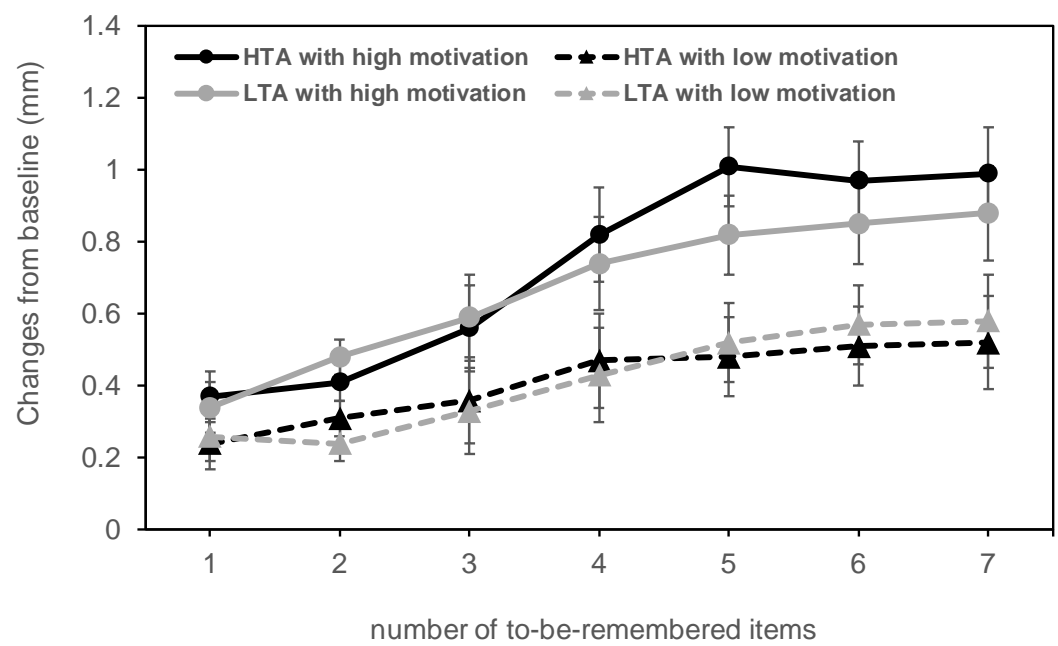

(b)

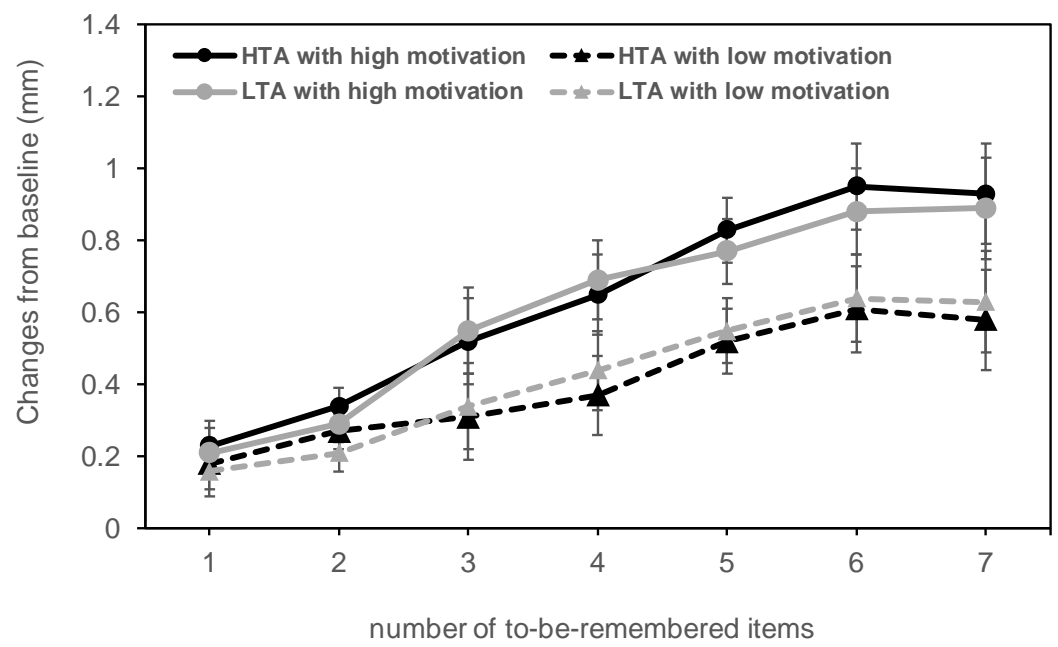

(c)

Figure 2. Baseline-corrected pupil dilation according to the levels of trait anxiety, motivational states, and cognitive loads. Error bars denote standard errors of the mean. (a) Pupil responses on low cognitive load condition; (b) pupil responses on moderate cognitive load condition; (c) pupil responses on high cognitive load condition. 
to the LTA group. At each cognitive load level, the group differences between the HTA and LTA were emerged in low and moderate load, $F(1,132)=6.65$, 8.441; $\mathrm{p}=0.0110,0.00043$, respectively, but not in high load, $\mathrm{F}(1,132)=1.13, \mathrm{p}$ $=0.290$. These results confirm that high levels of trait anxiety (along with a situational demand) drive additional cognitive effort to achieve a task goal, as ACT predicts. Still, when cognitive load is fairly severe (i.e., high load), group differences between the HTA and LTA disappear presumably due to the ceiling effects.

\section{Discussion}

The present study examined whether a detrimental effect of trait anxiety on executive control could negatively influence the maintenance of WM contents on a specific variation of complex span task. In addition, the modulatory effect of motivational states on the relationship between trait anxiety and cognitive task performance was also tested using the pupillometry technique. We observed that, in low motivational states, high trait-anxious individuals selectively performed worse, compared to those low in trait anxiety, in the low to moderateload conditions. There is a clear trend of steeper decline found in the high traitanxiety group as the cognitive load increases. In contrast to that, no group differences in task performance were observed in high motivational states.

As for the measure of motivational states, high and low trait-anxious individuals differed selectively in high motivation condition with low to moderate cognitive load. In such situations, high trait anxious individuals can be more motivated (through apprehension about their task performance) and invest more cognitive effort on the task at hand, as ACT predicts. Still, when cognitive load is fairly severe (i.e., high load), group differences between the HTA and LTA disappear presumably due to the ceiling effects.

Our results point to a modulatory effect of time-consuming nature of cognitive load and motivational states on the association of anxiety and WM maintenance. The detrimental effect of anxiety on attentional control has been demonstrated in previous studies, but findings concerning the relation between anxiety and WM maintenance, especially verbal domain, are mixed [30] [31]. The current study demonstrates that the time-consuming cognitive load was crucial for describing the association of anxiety and WM maintenance. Eysenck and Calvo [32] suggest that task difficulty is one crucial factor for detecting the detrimental effect of anxiety on cognitive task performance. Our findings point out that one element of task difficulty is the severity of the time limit on the available time to refresh WM items as TBRS theory postulates. Furthermore, the current study demonstrates that the relationship between trait anxiety and cognitive performance is clearly moderated by participants' motivational states. Hayes et al. [21] considered systematically this subject using behavioral performances and preferences to intentional or incidental learning. Our contribution to this subject is that, by using psychophysiological measure of cognitive effort or motivation, the current study provides direct support simultaneously for anxiety-related 
cognitive inefficiency and compensatory effort hypothesis derived from ACT.

WM performances were selectively related to trait anxiety but not to state anxiety. Previous studies demonstrated that state anxiety could strengthen stimulus-driven attention, and would not affect executive control [7]. In line with this finding, in the current study the levels of state anxiety were not predictive of performance on the continuous processing span task which depends heavily on executive control. Given that the scores on STAI-S were somewhat low irrespective of the level of trait anxiety, it is possible that intense state anxiety might affect executive processes under stressful situations. This possibility is becoming further elucidated in recent studies [33] [34].

To the best of our knowledge, this is the first investigation based on the TBRS theory of the detrimental effect of anxiety on WM tasks in conjunction with the motivational state. However, our findings have some limitations. First, even though TBRS theory assumes that the between-task (WM and processing tasks) shifting is crucial, focus shifting "between" WM contents is also time-consuming [35] [36]. Recently, Hoshino and Tanno [37] demonstrated that high levels of trait anxiety disrupts efficient attentional control between retained WM contents under the absence of external distracter. Therefore, further studies are needed to elucidate the role of focus shifting in performing the WM tasks, and especially the effect of anxiety on this reflective process. Secondly, we did not assess participants' state of apprehension or worry. In this study, state anxiety, a closely related concept of worry, showed no relations to WM performance irrespective of task goals (motivation) and demands (cognitive load). admittedly, our task goal settings used as an experimental manipulation did not explicitly induce worry, but increased motivational states. Hence, more elaborative manipulation and measure(s) of worry are needed to examine the anxiety-driven compensatory effort process in conforming strictly to the framework of ACT. Thirdly, although the HTA and LTA groups were equivalent in WM performance in high motivational states, measures of general intelligence or other personality traits were not included in our study. As a consequence, baseline general cognitive ability and personality traits relating to anxiety were not controlled. Future studies investigating the association of anxiety and cognition should include these variables for controlling extraneous variables.

Finally, implication for clinical practices is discussed. It is worth noting that the decrement of WM functioning in high-anxiety individuals would be related to their poorer performance on higher-order cognition, such as reading comprehension [38], reasoning/inference [39] [40], or math exam [10]. High-anxious individuals can be suffered from distractibility and difficulty concentrating on task at hand. Therefore, executive function trainings including attention and WM are now emerging treatment for the cognitive dysfunction(s) in anxiety [41]. Unfortunately, executive function trainings do not have precise methodology concerning attention control toward WM or mental representations such as thought content. Predicting and overcoming the negative impact of anxiety on higher-order cognition from lower level executive processes (i.e., attention, and 
working memory) might be one promising direction for further establishing the clinical methodology of cognitive trainings for individuals suffering from anxiety.

\section{Acknowledgements}

This study was financially supported by a grant-in-aid for JSPS fellows.

\section{References}

[1] Spielberger, C.D., Gorsuch, R. and Lushene, R. (1970) STAI: Manual for the State-Trait Anxiety Inventory. TEA Ediciones, S.A., Madrid.

[2] Eysenck, M.W. and Derakshan, N. (2011) New Perspectives in Attentional Control Theory. Personality and Individual Differences, 50, 955-960. https://doi.org/10.1016/j.paid.2010.08.019

[3] Eysenck, M.W., Derakshan, N., Santos, R. and Calvo, M.G. (2007) Anxiety and Cognitive Performance: Attentional Control Theory. Emotion, 7, 336-353. https://doi.org/10.1037/1528-3542.7.2.336

[4] Baddeley, A. (2007) Working Memory, Thought, and Action. Oxford University Press, Oxford. https://doi.org/10.1093/acprof:oso/9780198528012.001.0001

[5] Cowan, N. (2005) Working Memory Capacity. Psychology Press. Hove, East Sussex. https://doi.org/10.4324/9780203342398

[6] Hirsch, C.R. and Mathews, A. (2012) A Cognitive Model of Pathological Worry. Behaviour Research and Therapy, 50, 636-646. https://doi.org/10.1016/j.brat.2012.06.007

[7] Pacheco-Unguetti, A.P., Acosta, A., Callejas, A. and Lupiáñez, J. (2010) Attention and Anxiety: Different Attentional Functioning under State and Trait Anxiety. Psychological Science, 21, 298-304. https://doi.org/10.1177/0956797609359624

[8] Ansari, T.L. and Derakshan, N. (2010) Anxiety Impairs Inhibitory Control But Not Volitional Action Control. Cognition and Emotion, 24, 241-254. https://doi.org/10.1080/02699930903381531

[9] Derakshan, N., Ansari, T.L., Shoker, L., Hansard, M.E. and Eysenck, M.W. (2009) Anxiety, Inhibition, Efficiency, and Effectiveness: An Investigation Using the Antisaccade Task. Experimental Psychology, 56, 48-55. https://doi.org/10.1027/1618-3169.56.1.48

[10] Ashcraft, M.H. and Kirk, E.P. (2001) The Relationships among Working Memory, Math Anxiety, and Performance. Journal of Experimental Psychology: General, 130, 224-237. https://doi.org/10.1037/0096-3445.130.2.224

[11] Calvo, M.G., Ramos, P.M. and Estevez, A. (1992) Test Anxiety and Comprehension Efficiency: The Role of Prior Knowledge and Working Memory Deficits. Anxiety, Stress, \& Coping, 5, 125-138. https://doi.org/10.1080/10615809208250492

[12] Darke, S. (1988) Anxiety and Working Memory Capacity. Cognition and Emotion, 2, 145-154. https://doi.org/10.1080/02699938808408071

[13] Sorg, B.A. and Whitney, P. (1992) The Effect of Trait Anxiety and Situational Stress on Working Memory Capacity. Journal of Research in Personality, 26, 235-241.

[14] Daneman, M. and Carpenter, P.A. (1980) Individual Differences in Working Memory and Reading. Journal of Verbal Learning and Verbal Behavior, 19, 450-466.

[15] Turner, M.L. and Engle, R.W. (1989) Is Working Memory Capacity Task Dependent? Journal of Memory and Language, 28, 127-154. 
[16] Barrouillet, P., Bernardin, S. and Camos, V. (2004) Time Constraints and Resource Sharing in Adults' Working Memory Spans. Journal of Experimental Psychology. General, 133, 83-100. https://doi.org/10.1037/0096-3445.133.1.83

[17] Barrouillet, P., Bernardin, S., Portrat, S., Vergauwe, E. and Camos, V. (2007) Time and Cognitive Load in Working Memory. Journal of Experimental Psychology: Learning Memory \& Cognition, 33, 570-585. https://doi.org/10.1037/0278-7393.33.3.570

[18] Cowan, N. (1999) An Embedded Process Model of Working Memory. In: Miyake, A. and Shah, P., Eds., Models of Working Memory: Mechanisms of Active Maintenance and Executive Control, Cambridge University Press, Cambridge, 62-101. https://doi.org/10.1017/CBO9781139174909.006

[19] Raye, C.L., Johnson, M.K., Mitchell, K.J., Greene, E.J. and Johnson, M.R. (2007) Refreshing: A Minimal Executive Function. Cortex, 43, 135-145.

[20] Barrouillet, P. and Camos, V. (2010) Working Memory and Executive Control: A Time-Based Resource-Sharing Account. Psychologica Belgica, 50, 353-382. https://doi.org/10.5334/pb-50-3-4-353

[21] Hayes, S., MacLeod, C. and Hammond, G. (2009) Anxiety-Linked Task Performance: Dissociating the Influence of Restricted Working Memory Capacity and Increased Investment of Effort. Cognition and Emotion, 23, 753-781. https://doi.org/10.1080/02699930802131078

[22] Heitz, R.P., Schrock, J.C., Payne, T.W. and Engle, R.W. (2008) Effects of Incentive on Working Memory Capacity: Behavioral and Pupillometric Data. Psychophysiology, 45, 119-129.

[23] Kahneman, D. and Beatty, J. (1966) Pupil Diameter and Load on Memory. Science, 154, 1583-1585. https://doi.org/10.1126/science.154.3756.1583

[24] Porter, G., Troscianko, T. and Gilchrist, I.D. (2007) Effort during Visual Search and Counting: Insights from Pupillometry. Quarterly Journal of Experimental Psychology, 60, 211-229. https://doi.org/10.1080/17470210600673818

[25] Sibley, C., Coyne, J. and Baldwin, C. (2011) Pupil Dilation as an Index of Learning. Proceedings of the Human Factors and Ergonomics Society 55 th Annual Meeting, 55, 237-241. https://doi.org/10.1177/1071181311551049

[26] Spielberger, C.D., Gorsuch, R.L., Lushene, R., Vagg, P.R. and Jacobs, G.A. (1983) Manual for the State-Trait Anxiety Inventory. Consulting Psychologists Press, Palo Alto, CA.

[27] Shimizu, H. and Imae, K. (1981) Development of the Japanese Edition of the Spielberger State-Trait Anxiety Inventory (STAI) for Student Use. Japanese Journal of Educational Psychology, 29, 62-67.

[28] Conway, A.R., Kane, M.J., Bunting, M.F., Hambrick, D.Z., Wilhelm, O. and Engle, R.W. (2005) Working Memory Span Tasks: A Methodological Review and User's Guide. Psychonomic Bulletin \& Review, 12, 769-786.

https://doi.org/10.3758/BF03196772

[29] Papesh, M.H. and Goldinger, S.D. (2012) Pupil-Blahmetry: Cognitive Effort in Speech Planning Reflected by Pupil Dilation. Attention, Perception, \& Psychophysics, 74, 754-765. https://doi.org/10.3758/s13414-011-0263-y

[30] Eysenck. M.W., Payne, S. and Derakshan, N. (2005) Trait Anxiety, Visuospatial Processing and Working Memory. Cognition and Emotion, 19, 1214-1228. https://doi.org/10.1080/02699930500260245

[31] Walkenhorst, E. and Crowe, S.F. (2009) The Effect of State Worry and Trait Anxiety on Working Memory Processes in a Normal Sample. Anxiety, Stress \& Coping, 22, 
167-187. https://doi.org/10.1080/10615800801998914

[32] Eysenck, M.W. and Calvo, M.G. (1992) Anxiety and Performance: The Processing Efficiency Theory. Cognition and Emotion, 6, 409-434. https://doi.org/10.1080/02699939208409696

[33] Edwards, M.S., Edwards, E.J. and Lyvers, M. (2017) Cognitive Trait Anxiety, Stress and Effort Interact to Predict Inhibitory Control. Cognition \& Emotion, 31, 671686.

[34] Vytal, K.E., Cornwell, B.R., Letkiewicz, A.M., Arkin, N.E. and Grillon, C. (2013) The Complex Interaction between Anxiety and Cognition: Insight from Spatial and Verbal Working Memory. Frontiers in Hum Neuroscience, 7, 93. https://doi.org/10.3389/fnhum.2013.00093

[35] Garavan, H. (1998) Serial Attention within Working Memory. Memory \& Cognition, 26, 263-276. https://doi.org/10.3758/BF03201138

[36] Oberauer, K. (2002) Access to Information in Working Memory: Exploring the Focus of Attention. Journal of Experimental Psychology. Learning, Memory, and Cognition, 28, 411-421. https://doi.org/10.1037/0278-7393.28.3.411

[37] Hoshino, T. and Tanno, Y. (2016) Trait Anxiety and Impaired Control of Reflective Attention in Working Memory. Cognition and Emotion, 30, 369-377. https://doi.org/10.1080/02699931.2014.993597

[38] Calvo, M.G. and Eysenck, M. (1996) Phonological Working Memory and Reading in Test Anxiety. Memory, 4, 289-306. https://doi.org/10.1080/096582196388960

[39] MacLeod, C. and Donnellan, A.M. (1992) Individual Differences in Anxiety and the Restriction of Working Memory Capacity. Personality and Individual Differences, $15,163-173$.

[40] Markham, R. and Darke, S. (1991) The Effect of Anxiety on Verbal and Spatial Task Performance. Australian Journal of Psychology, 43, 107-111. https://doi.org/10.1080/00049539108259108

[41] Bomyea, J. and Amir, N. (2011) The Effect of an Executive Functioning Training Program on Working Memory Capacity and Intrusive Thoughts. Cognitive Therapy \& Research, 35, 529-535. https://doi.org/10.1007/s10608-011-9369-8

\section{Submit or recommend next manuscript to SCIRP and we will provide best} service for you:

Accepting pre-submission inquiries through Email, Facebook, LinkedIn, Twitter, etc. A wide selection of journals (inclusive of 9 subjects, more than 200 journals)

Providing 24-hour high-quality service

User-friendly online submission system

Fair and swift peer-review system

Efficient typesetting and proofreading procedure

Display of the result of downloads and visits, as well as the number of cited articles

Maximum dissemination of your research work

Submit your manuscript at: http://papersubmission.scirp.org/

Or contact jbbs@scirp.org 